\title{
The Potential Role of Thioctic Acid in the Attenuation of Doxorubicin Induced-Cardiotoxicity
}

\author{
Hayder M Al kuraishy ${ }^{1 *}$, Reem G Hussein ${ }^{2}$ and Ali I Al Gareeb ${ }^{1}$ \\ ${ }^{1}$ Department of Pharmacology, College of Medicine Al mustansiriya University, Iraq \\ ${ }^{2}$ Department of Pharmacology, Al-Yarmook University, Iraq
}

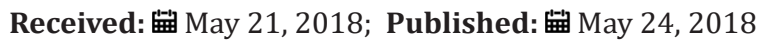

*Corresponding author: Hayder M Al kuraishy, Professor in Department Of Pharmacology, Toxicology and Medicine, College of Medicine Al mustansiriya University, P.O. Box 14132 Iraq /Baghdad

\section{Introduction}

During 1950s in Italy a soil sample was isolated and found new strains of Streptomyces peucetius bacteria from which a new antibiotic was extracted with potent effect against murine tumors, this antibiotic named daunorubicin [1]. In 1960s, a clinical trial was done on the drug and result a successful in treating acute leukemia and lymphoma, and finally a new antibiotic was discovered which named adriamycin which change to doxorubicin. Doxorubicin has a potent antitumor activity more than daunorubicin with a higher therapeutic index [2]. In 1967 the cardiotoxicity due to danurubicin was approved, the greatest risk of doxorubicin-induced toxicity is cardiotoxicity, so administration of doxorubicin should be doselimited [3]. Doxorubicin changes the structure and function of cardiomyocytes, the genes that cause this are the brain natriuretic peptide (BNP) and atrial natriuretic peptide (ANP) which are highly expressed in doxorubicin-induce cardiotoxicity, so; these two genes are responsible for cardiac hypertrophy [4]. The molecular mechanism behind this event involves formation of oxygen free radicals and iron oxidation. Since doxorubicin known to affect multiple biomarkers, the assessment of troponins and specific natriuretic peptides (pro BNP and DNP) is believed to predict doxorubicin-induced cardiotoxicity in early stages [5].

\section{Mechanism of Doxorubicin-Induced Cardiotoxicity}

Oxidative Stress: Doxorubicin-induced acute cardiotoxicity is result from oxidative stress and free radical production which induced by high dose of doxorubicin that initiating oxidative myocardial injury due to conversion of doxorubicin by intracellular enzymes into semiquinone free radical [6]. The heart is most susceptible organ to damage by free radicals due to low activities of antioxidant enzyme such as (superoxide dismutase, glutathione, and catalase enzymes) as a result, the accumulation of free radicals that lead to lipid peroxidation, mitochondrial membrane damage, injury of nucleic acid, and destruction of myocardium endoplasmic reticulum [7].

Mitochondrial Damage: Mitochondria considered as the most progressively and extensively injured in doxorubicin induced cardiotoxicity because of the cationic drug doxorubicin which accumulated in the inner layer of the mitochondrial that forming a nearly-irreversible compound with cardiolipin [8]. The protein that needs cardiolipin coupled to work correctly, and since doxorubicin disrupt the cardiolipin protein interface and forming more superoxide [9]. Moreover, transport carnitine is extremely affected by doxorubicin that contributes to the mitochondrial dysfunction [10]. It is quite acceptable that these actions will disrupt mitochondrial metabolism, since mitochondria responsible for production of $90 \%$ of the Adenosine triphosphate (ATP) utilized by cardiomyocytes so, many ultrastructural pathologic changes will take place such as mitochondrial swelling and myelin figures within the mitochondria [11].

Doxorubicin-Iron Complex: Doxorubicin iron complex had been recognized that doxorubicin has a strong affinity to iron and this complex increase the lipid peroxidation through its interactions. In the presence of unbound iron atom, doxorubicin will encourage to form a cycle of free radical production, also doxorubicin metabolite form doxorubicinol which have the ability to interact with thiol groups of cell membrane proteins [12].

Apoptosis: The oxidative stress that evoked by doxorubicin can initiates the apoptotic signal leading to cardiomyocyte apoptosis also; the extrinsic and intrinsic apoptotic-pathways will be involved. Noticeably, doxorubicin induces apoptosis indirectly via production of free radicals [13]. 
Intracellular Calcium Dysregulation: Doxorubicin-induced cardiotoxicity can be augmented by increase the level of intracellular calcium and the dysregulation of intracellular calcium that generate free radicals [14]. The redox oxygen species and $\mathrm{H}_{2} \mathrm{O}_{2}$ generated also change the homeostasis of calcium in a multiple muscle cell types by disturbance of normal sarcoplasmic reticulum function. This is accomplished by inhibiting the $\mathrm{Ca}^{2+}$ ATPase pump [15] Doxorubicin increases the release of calcium from the sarcoplasmic reticulum by increasing the chance of its channel's open state. Additionally, doxorubicin inhibits the sodium calcium exchanger channel inside sarcolemma that enhances the activity of the L-type calcium channel [16].

Neutrophils Activation: Myocardial damage also caused by neutrophils activation which releases myeloperoxidase and IL-17 so; these mediators regarded as potent indicators of cardiotoxicity [17]. Thioctic acid also named lipoic acid is a fatty acid compound that contains sulfur in its structure. Lipoic acid acts as an antioxidant and co-enzyme in the body so it called universal antioxidant because of the ability to modulate different kind of free radicals. Several studies showed that thioctic acid improves several conditions in the body that caused by free radicals, for example heart disease, cancer and other disorders which related with inflammation and aging [18]. The oxidized thioctic acid and reduced thioctic acid forms a potent redox couple that has a potent reduction potential so they consider as a potent antioxidants [19]. Truly, both thioctic acid and reduced thioctic acid scavenge different type of free radical such as hydroxyl radicals and hypochlorous acid, but the thioctic acid only cans scavenge singlet oxygen [20]. Furthermore, thioctic acid stimulates other endogenous antioxidants (e.g. vitamins $\mathrm{C}$ and E) and neutralizing many oxygen species [21]. Moreover, thioctic acid chelate redox-active metals in vitro and in vivo. The oxidizing form and reducing form bind with a number of metal ions, but in different properties according to the metal types [22]. Thioctic acid enters in recycle and stimulates many transcription factors, such these transcription factors that responsible for the production of GSH which is Nrf2. Under stress when the amount of free radical increase Nrf2 amount will decrease, at this moment lipoic acid will protect Nrf2 from influence by oxidative stress by its action as anti-oxidant [23]. Thioctic acid has been reported to attenuate and prevent myocardial damage during ischemic-reperfusion injury and anthracyline induced cardiotoxicity [24].

Thioctic acid has the ability to decrease serum BNP and cardiac hypertrophy by attenuation of mRNA and protein levels of $\mathrm{C}$ / EBP $\beta$ which is responsible for cardiomyocyte hypertrophy. Also, thioctic acid decrease BNP serum levels through acting directly on scavenging free radicals, increasing the activity of natural antioxidant, reduction of oxidative stress and inflammation [25]. Moreover, thioctic acid decreases the level of caspase- 3 due to antioxidant, anti-inflammatory and anti-apoptotic properties against doxorubicin toxicity in rats [26]. Additionally thioctic acid causes significant increases in serum glutathione peroxidase level due to its potent antioxidant activity [27]. As well, thioctic acid illustrated a significant decrease in cardiac troponin I serum levels due to its antioxidant properties which modulate the oxidative stress and enhancement of endogenous glutathione [28]. In addition, thioctic acid has a significant effect in reduction of lipid peroxidase due to significant anti-oxidative and anti-inflammatory properties [29]. Over and above, thioctic acid led to a significant decrease in serum malondialdehyde serum levels as in many experimental contemporary studies that showed a significant effect of thioctic acid on the reduction of MDA serum levels during acute doxorubicin induced-cardiotoxicity in rat due to the potential antioxidant activity [30].

Furthermore, many experimental studies illustrated a significant decrease in TNF- $\alpha$ serum levels in rats that pre treated with thioctic acid due to reduction of LPS-stimulated release of inflammatory cytokines, such as tumor necrosis factor alpha (TNF- $\alpha$ ), interleukin (IL-1), (IL-1 $\beta$ ) and IL-6 [31]. Therefore, thioctic acid produced significant cardio-protection due to different mechanisms. Thioctic acid is a potent co-factor for mitochondrial dehydrogenase so; it preserves mitochondrial function during cardiac injury through reduction of ATP hydrolysis, augmentation of ATP production and normalization of cardiomyocte PH [32]. Thioctic acid also prevents myocardial necrosis and apoptosis via inhibition of ischemic-reperfusion damage which triggered by oxidative stress [33]. The molecular mechanism of thioctic acid in attenuation of doxorubicin-induced cardiotoxicity is so complicated. During cardiotoxicity, proapoptotic gene is activated that trigger protein kinase MAP, nuclear factor-kB and TNF- $\alpha$ thereby apoptosis will be induced. The extra-cellular signals of protein kinase are $p 38 \alpha / \beta$ kinase, regulated kinase ERK1/2 and terminal kinase c-Jun-NH2 [34]. Regulated kinase ERK1/2 is concerned with cell survival while terminal kinase c-Jun-NH2 is involved in induction of apoptosis thus; ERK1/2 antagonist led to significant apoptosis since; ERK1/2 has anti-apoptotic property. Therefore, the molecular effect of thioctic acid during doxorubicininduced cardiotoxicity is activation of anti apoptotic pathway and inhibition of apoptotic pathway [35].

\section{Conclusion}

Thioctic acid illustrated a significant cardio-protection during doxorubicin induced-cardiotoxicity through modulation of oxidative stress and anti-oxidant potential.

\section{References}

1. Hayder M Alkuraishy, Ali I Al Gareeb, Hany A Al hussaniy (2017) Doxorubicin-Induced Cardiotoxicity: Molecular Mechanism and Protection by Conventional Drugs and Natural Products. International Journal of Clinical Oncology and Cancer Research 2(2): 31-44.

2. Huang S, Zhang Y, Zhong J, Pan Y, Cai S, et al. (2018) Toxicological profile and safety pharmacology of a single dose of fibroblast activation protein$\alpha$-based doxorubicin prodrug: in-vitro and in-vivo evaluation. Anticancer Drugs 29(3): 253-261.

3. Chang VY, Wang JJ (2018) Pharmacogenetics of Chemotherapy-Induced Cardiotoxicity. Curr Oncol Rep 20(7): 52. 
4. Pugazhendhi A, Edison TNJI, Velmurugan BK, Jacob JA, Karuppusamy I (2018) Toxicity of Doxorubicin (Dox) to different experimental organ systems. Life Sci 200: 26-30.

5. Yu J, Wang C, Kong Q Wu X, Lu JJ, et al. (2018) Recent progress in doxorubicin-induced cardiotoxicity and protective potential of natural products. Phytomedicine 40: 125-139.

6. Maayah ZH, Abdelhamid G, Elshenawy OH, El Sherbeni AA, Althurwi HN, et al. (2017) The Role of Soluble Epoxide Hydrolase Enzyme on Daunorubicin-Mediated Cardiotoxicity. Cardiovasc Toxicol 18(3): 268283.

7. Benzer F, Kandemir FM, Ozkaraca M, Kucukler S, Caglayan C (2018) Curcumin ameliorates doxorubicin-induced cardiotoxicity by abrogation of inflammation, apoptosis, oxidative DNA damage, and protein oxidation in rats. J Biochem Mol Toxicol 32(2).

8. Yin J, Guo J, Zhang Q Cui L, Zhang L, et al. (2018) Doxorubicin induced mitophagy and mitochondrial damage is associated with dysregulation of the PINK1/parkin pathway. Toxicol In Vitro 51: 1-10.

9. Pereira GC, Pereira SP, Tavares LC, Carvalho FS, Magalhães Novais S, et al. (2016) Cardiac cytochrome c and cardiolipin depletion during anthracycline-induced chronic depression of mitochondrial function. Mitochondrion 30: 95-104.

10. Mustafa HN, Hegazy GA, Awdan SAE, Abdel Baset M (2017) Protective role of CoQ10 or L-carnitine on the integrity of the myocardium in doxorubicin induced toxicity. Tissue Cell 49(3): 410-426.

11. Gorini S, De Angelis A, Berrino L, Malara N, Rosano G, et al. (2018) Chemotherapeutic Drugs and Mitochondrial Dysfunction: Focus on Doxorubicin, Trastuzumab, and Sunitinib. Oxid Med Cell Longev 2018: 7582730 .

12. Al Kuraishy HM, Al Gareeb AI (2016) Potential Effects of Pomegranate on Lipid Peroxidation and Pro-inflammatory Changes in Daunorubicin induced Cardiotoxicity in Rats. Int J Prev Med 7: 85.

13. Wang Y, Lei T, Yuan J, Wu Y, Shen X, et al. (2018) GCN2 deficiency ameliorates doxorubicin-induced cardiotoxicity by decreasing cardiomyocyte apoptosis and myocardial oxidative stress. Redox Biol 17: $25-34$.

14. Agustini FD, Arozal W, Louisa M, Siswanto S, Soetikno V, et al. (2016) Cardioprotection mechanism of mangiferin on doxorubicin induced rats: Focus on intracellularcalcium regulation. Pharm Biol 54(7): 1289-1297.

15. Burke BE, Mushlin PS, Cusack BJ, Olson SJ, Gambliel HA (2002) Decreased sensitivity of neonatal rabbit sarcoplasmic reticulum to anthracycline cardiotoxicity. Cardiovasc Toxicol 2(1): 41-51.

16. Hanna AD, Lam A, Thekkedam C, Willemse H, Dulhunty AF, et al. (2017) The Anthracycline Metabolite Doxorubicinol Abolishes RyR2 Sensitivity to Physiological Changes in Luminal $\mathrm{Ca}^{2+}$ through an Interaction with Calsequestrin. Mol Pharmacol 92(5): 576-587.

17. Schofield ZV, Woodruff TM, Halai R, Wu MC, Cooper MA (2013) Neutrophils a key component of ischemia-reperfusion injury. Shock 40(6): 463-470.

18. Hayder M Al Kuraishy, Reem G Hussein (2017) Caspase-3 Levels (CASP-3) in Doxorubicin Induced-Cardiotoxicity: Role of Metformin Pretreatment. Research Journal of Oncology 1(1): 4

19. Hayder M Al kuraishy, Ali I Al Gareeb (2016) Co-administration effects of $\alpha$-lipoic acid and nucleo CMP on arousal and sensory cortical activity. J Young Pharm 8(1): 12-17.

20. Mohammadi V, Khorvash F, Feizi A, Askari G (2018) Does Alpha lipoic Acid Supplementation Modulate Cardiovascular Risk Factors in Patients with Stroke? A Randomized, Double blind Clinical Trial. Int J Prev Med 9: 34 .

21. Shen W, Hao J, Feng Z, Tian C, Chen W, et al. (2011) Lipoamide or lipoic acid stimulates mitochondrial biogenesis in 3T3-L1 adipocytes via the endothelial NO synthase-cGMP-protein kinase G signalling pathway. Br J Pharmacol 162(5): 1213-1224.

22. Huk Kolega H, Skibska B, Kleniewska P, Piechota A, Michalski Ł, et al. (2011) Role of lipoic acid in health and disease. Pol Merkur Lekarski 31(183): 183-185.

23. Lv C, Maharjan S, Wang Q Sun Y, Han X, et al. (2017) $\alpha$-Lipoic Acid Promotes Neurological Recovery After Ischemic Stroke by Activating the Nrf2/HO-1 Pathway to Attenuate Oxidative Damage. Cell Physiol Biochem 43(3): 1273-1287.

24. Shaafi S, Afrooz MR, Hajipour B, Dadadshi A, Hosseinian MM, et al. (2011) Anti-oxidative effect of lipoic Acid in spinal cord ischemia/reperfusion. Med Princ Pract 20(1): 19-22.

25. Zou J, Gan X, Zhou H, Chen X, Guo Y (2015) Alpha-lipoic acid attenuates cardiac hypertrophy via inhibition of C/EBP $\beta$ activation. Mol Cell Endocrinol 399: 321-329.

26. Dörsam B, Göder A, Seiwert N, Kaina B, Fahrer J (2015) Lipoic acid induces p53-independent cell death in colorectal cancer cells and potentiates the cytotoxicity of 5-fluorouracil. Arch Toxicol 89(10): 18291846.

27. Lu M, Bai J, Wei F, Xu B, Sun Q, et al. (2017) ects of alpha-lipoic acid supplementation on growth performance, antioxidant capacity and biochemical parameters for ammonia-exposed broilers. Anim Sci J 88(8): 1220-1225.

28. Marfella R, Barbieri M, Sardu C, Rizzo MR, Siniscalchi M, et al. (2016) Effects of $\alpha$-lipoic acid therapy on sympathetic heart innervation in patients with previous experience of transient takotsubo cardiomyopathy. J Cardiol 67(2): 153-161.

29. Piechota Polanczyk A, Zielińska M, Piekielny D, Fichna J (2016) The influence of lipoic acid on caveolin-1-regulated antioxidative enzymes in the mouse model of acute ulcerative colitis. Biomed Pharmacother 84: $470-475$.

30. Derosa G, D Angelo A, Romano D, Maffioli P (2016) A Clinical Trial about a Food Supplement Containing $\alpha$-Lipoic Acid on Oxidative Stress Markers in Type 2 Diabetic Patients. Int J Mol Sci 17(11).

31. Chang P, Liu J, Yu Y, Cui SY, Guo ZH (2017) Alpha-Lipoic Acid Suppresses Extracellular Histone-Induced Release of the Infammatory Mediator Tumor Necrosis Factor- $\alpha$ by Macrophages. Cell Physiol Biochem 42(6): 2559-2568.

32. Cao Z, Tsang M, Zhao H, Li Y (2003) Induction of endogenous antioxidants and phase 2 enzymes by alpha-lipoic acid in rat cardiac H9C2 cells: protection against oxidative injury. Biochem Biophys Res Commun 310(3): 979-985.

33. KO KM, Yiu HY (2001) Schisandrin B modulates the ischemiareperfusion induced changes in non-enzymatic antioxidant levels in isolated-perfused rat hearts. Mol Cell Biochem 220(1-2): 141-147.

34. Yoo TH, Lee JH, Chun HS, Chi SG (2013) $\alpha$-Lipoic acid prevents p53 degradation in colon cancer cells by blocking $N F-\kappa B$ induction of RPS6KA4. Anticancer Drugs 24(6): 555-565.

35. Yang L, Wen Y, Lv G, Lin Y, Tang J, et al. (2017) $\alpha$-Lipoic acid inhibits human lung cancer cell proliferation through Grb2-mediated EGFR downregulation. Biochem Biophys Res Commun 494(1-2): 325-331. 
(C) (i) This work is licensed under Creative

To Submit Your Article Click Here:

Submit Article

DOI: 10.32474/OAJOM.2018.01.000120

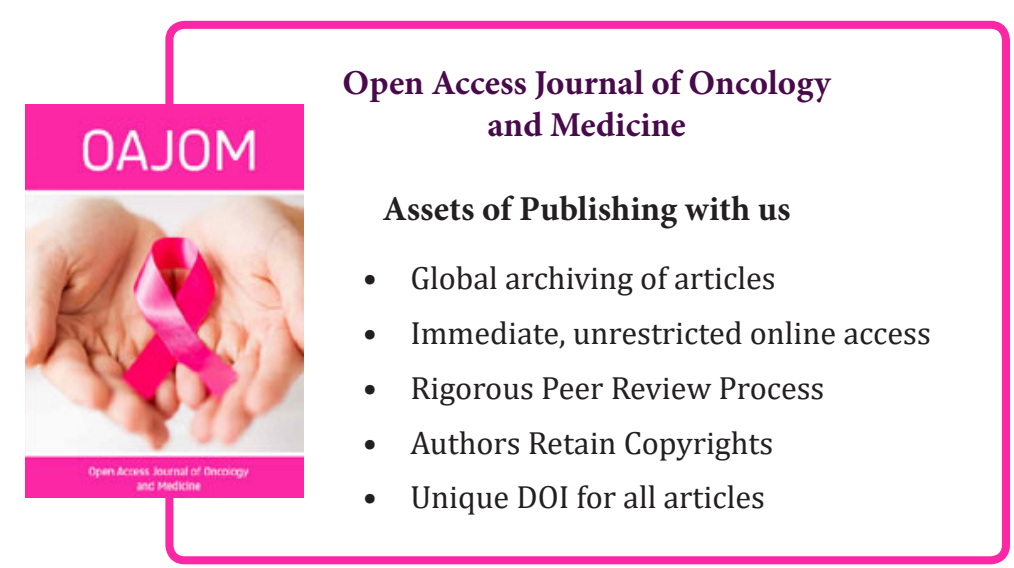

